\title{
Asymptomatic Strongyloides stercoralis Hyperinfection in an Alcoholic Patient with Intense Anemia
}

Author(s) :M. C. A. Teixeira, E. J. Inês, F. T. F. Pacheco, R. K. N. R. Silva, A. V. Mendes, E. V. Adorno, F. M. Lima, and N. M. Soares

Source: Journal of Parasitology, 96(4):833-835. 2010.

Published By: American Society of Parasitologists

DOI: http://dx.doi.org/10.1645/GE-2358.1

URL: http://www.bioone.org/doi/full/10.1645/GE-2358.1

BioOne (www.bioone.org) is a nonprofit, online aggregation of core research in the biological, ecological, and environmental sciences. BioOne provides a sustainable online platform for over 170 journals and books published by nonprofit societies, associations, museums, institutions, and presses.

Your use of this PDF, the BioOne Web site, and all posted and associated content indicates your acceptance of BioOne's Terms of Use, available at www.bioone.org/page/terms_of_use.

Usage of BioOne content is strictly limited to personal, educational, and non-commercial use. Commercial inquiries or rights and permissions requests should be directed to the individual publisher as copyright holder. 


\section{Asymptomatic Strongyloides stercoralis Hyperinfection in an Alcoholic Patient with Intense Anemia}

M. C. A. Teixeira, E. J. Inês, F. T. F. Pacheco, R. K. N. R. Silva, A. V. Mendes ${ }^{\star}$, E. V. Adorno, F. M. Lima, and N. M. Soares, Departmento de Análises Clínicas e Toxicológicas, Universidade Federal da Bahia, Bahia, Brazil 40170-115; 'Escola Bahiana de Medicina e Saúde Pública, Bahia, Brazil 40050-420 and Hospital São Rafael, Monte Tabor, Bahia, Brazil 41.256-900. e-mail: marciat@ufba.br

ABSTRACT: Strongyloides stercoralis infection is endemic in many tropical and subtropical areas. The parasite has the unusual ability to multiply inside the host due to the transformation of rhabditiform larvae into infective filariforms. Several studies have shown that chronic alcoholism is an important factor that predisposes to strongyloidiasis. The increased susceptibility to $S$. stercoralis infections seen in alcoholic individuals could be explained by their increased exposure to the parasite, malnutrition, breakdown of local immune responses, and/or alterations in intestinal barriers. Moreover, ethanol intoxication can elevate human endogenous corticosterone, which, in turn, suppresses $T$ cell function and increases the fecundity and survival of the parasite, mimicking the effect of worm ecdysteroides. Although chronic alcoholism is a risk factor for nematode infection, most cases of hyperinfection or dissemination are associated with the presence of hepatic cirrhosis or strongyloidiasis-related symptoms. The present study describes a case of $S$. stercoralis hyperinfection in a 51-yr-old male patient without gastrointestinal or pulmonary symptoms and with previous anemia and chronic alcoholism. He was not receiving glucocorticoid therapy and tested negative for HTLV and human immunodeficiency virus (HIV), but he had a history of alcohol addiction for more than $20 \mathrm{yr}$. Laboratory test results showed increased eosinophilia and a high immunoglobulin E (IgE) level, which may have temporarily protected the patient from dissemination of infection, but not prevented proliferation of the parasite, as shown by the large number of $S$. stercoralis larvae recovered using the Baermann method. Evaluation for strongyloidiasis should occur in alcoholics, especially in endemic areas, to prevent occult asymptomatic infections from progressing to life-threatening cases.

Strongyloides stercoralis infection is endemic in many tropical and subtropical areas (Concha et al., 2005), with frequencies above 10\% in immunocompetent Brazilian people (Rossi et al., 1993; Kobayashi et al., 1996; Machado and Costa-Cruz, 1998) and reaching 40-44\% in cirrhotic patients, mostly alcoholics (Gaburri et al., 1997; Oliveira et al., 2002).

The parasitic infection takes place when filariform larvae penetrate through the skin and migrate via the bloodstream to the lungs. Afte ascending the respiratory tract to the oropharynx, larvae are swallowed and reach the duodenal mucosal crypts to grow into parthenogenetic females. Thereafter, rhabditiform larvae hatch from the eggs and are excreted in feces. However, some larvae may transform into the filariform infective stage, resulting in internal and external autoinfection. Therefore, if not treated, the host may remain in a chronic carrier state.

The nematode usually causes an asymptomatic infection with a small rhabditoid larvae load in the feces (Rossi et al., 1993; Grove, 1996). Nevertheless, hyperinfection and dissemination can occur in high-risk groups, such as patients undergoing glucocorticoid therapy (Lemos et al, 2003), patients co-infected with HTLV (Carvalho and Porto, 2004) or HIV (Silva et al., 2005), patients with lymphoma (Safdar et al., 2004), and people with malnutrition (Dada-Adegbola and Bakare, 2004) or liver cirrhosis due to alcoholism (Gaburri et al., 1997).

Several studies have shown that chronic alcoholism is an important factor in susceptibility to strongyloidiasis (Gaburri et al., 1997; Oliveira et al., 2002; Zago-Gomes et al., 2002). It is hypothesized that the high predisposition to infection in this group could be associated with poor hygiene, leading to high exposure to pathogens, malnutrition, and alterations in either intestinal barriers or local immune responses (Gaburri et al., 1997; Zago-Gomes et al., 2002). The increased risk for infection with $S$. stercoralis in ethanol abusers appears to be specific, since this is not

\section{DOI: $10.1645 / G E-2358$.}

observed with other intestinal nematodes (Zago-Gomes et al., 2002). Although chronic alcoholism predisposes to $S$. stercoralis infection, most cases of hyperinfection and dissemination are associated with the presence of hepatic cirrhosis and/or pulmonary and gastrointestinal symptoms (Gaburri et al., 1997)

Different mechanisms may be responsible for the stimulation of hyperinfection in susceptible hosts, although corticosteroid therapy remains the most frequent risk factor. Acute or chronic ethanol administration in rats increases the plasma levels of adrenocorticotropic hormone and corticosterone, by both interfering with the hypothalamicpituitary-adrenal (HPA) axis and altering steroid metabolism in the liver (Ogilvie et al., 1998; László et al., 2001). Corticoids interfere in the host's immune response and also enhance the fecundity of $S$. stercoralis females and the transformation of rhabditiform into infective filariform larvae, exacerbating the autoinfection (Concha et al., 2005).

Strongyloides stercoralis infection is mostly controlled by the Th2 subset of CD4 lymphocytes, the production of interleukin (IL)-4, IL-5, IL-13, $\mathrm{IgE}$, and eosinophils. In addition, other host defense mechanisms, such as complement activation or Th1-related immune responses, may be involved in parasite killing (Szabo, 1999; Krolewiecki et al., 2001).

In the present study, we describe a $S$. stercoralis hyperinfection in an anemic patient with a history of chronic alcohol consumption and without gastrointestinal or pulmonary symptoms. All clinical data were collected by an infectious disease specialist. The patient's privacy and confidentiality were preserved and informed consent authorizing publication was obtained.

A 51-yr-old man presented to our laboratory for evaluation of previously identified anemia. At the time of his current admission, the patient had no clinical complaint of diarrhea, abdominal pain, productive cough, or fever, but did have symptoms related to anemia, such as shortness of breath and weakness.

Laboratory results showed a leukocyte count of $6.8 \times 10^{6} / \mathrm{mm}^{3}$, with a discrete eosinophilia $\left(595\right.$ eosinophils $/ \mathrm{mm}^{3}$ ) and normal platelet count $\left(309 \times 10^{3} / \mathrm{mm}^{3}\right)$. Normal liver (aspartate aminotransferase $22 \mathrm{IU} / \mathrm{L}$, alanine aminotransferase $13 \mathrm{IU} / \mathrm{L}$, total bilirubin $0.3 \mathrm{mg} / \mathrm{dl}$ ) and kidney (urea $22 \mathrm{mg} / \mathrm{dl}$; creatinine $0.8 \mathrm{mg} / \mathrm{dl}$ ) functions were observed.

The hematological analyses confirmed the presence of anemia. The patient had $1.6 \times 10^{6} / \mathrm{mm}^{3}$ erythrocytes, with low hematocrit $(13.6 \%)$ and hemoglobin $(4.5 \mathrm{~g} / \mathrm{dl})$ levels. Hematimetric values included a mean corpuscular volume of $85.2 \mathrm{fl}$, mean corpuscular hemoglobin of $26.6 \mathrm{pg}$, and mean corpuscular hemoglobin concentration of $31.2 \%$. The morphological analysis of the blood film was found to be markedly abnormal, showing hypochromia, anisocytosis, and poikilocytosis. Reduced levels of serum iron $(14 \mu \mathrm{g} / \mathrm{dl})$ and ferritin $(9.1 \mathrm{pg} / \mathrm{ml})$ were also observed. Direct Coombs test and glucose-6-phosphate dehydrogenase deficiency test were both negative. Vitamin B12 was $254 \mathrm{pg} / \mathrm{ml}$ and folic acid was $7.73 \mathrm{ng} / \mathrm{dl}$. The hemoglobin analysis demonstrated the sickle cell trait profile: A1 (57.5\%), S (38.5\%), A2 (3.8\%), and fetal hemoglobin (0. $2 \%)$.

Levels of serum immunoglobulins $\operatorname{IgA}(144 \mathrm{mg} / \mathrm{dl}), \operatorname{IgM}(207 \mathrm{mg} / \mathrm{dl})$, and $\operatorname{IgG}(1,040 \mathrm{mg} / \mathrm{dl})$ were normal, as were complement proteins C3 (134 mg/dl), C4 (21.2 mg/dl), and CH50 (101 U/CAE). Serum IgE level was extremely elevated, above $3,000 \mathrm{IU} / \mathrm{ml}$. Integrity of the cellular immune response was checked by the tuberculin reaction $(2.1 \mathrm{~cm})$, determination of the number of CD4 cells $(3,087 \mathrm{cell} / \mu \mathrm{l})$, and the $\mathrm{T}$ helper/ suppressor ratio (1.72).

The HTLV (I and II); HIV (1 and 2); hepatitis A, B, and C; Chagas disease; and syphilis antibody tests were negative. Lung fields were clear on the chest radiographs and digital electroencephalogram was also normal. 
Positive results were shown on testing for stool occult blood by phenolphthalein reduction with zinc. Further routine examination of feces by spontaneous sedimentation in water revealed several $S$. stercoralis rhabditiform larvae and also Giardia intestinalis cysts. Moreover, there were 910 larvae recovered per gram of stool concentrated by the Baermann method, mostly rhabditiform and a few filariform stages.

The patient received blood transfusions and was treated with conventional doses of ivermectin and tinidazole for $S$. stercoralis and $G$. intestinalis infection, respectively. The eosinophil count decreased after treatment and alcohol abstinence, and returned to normal levels, as did the hemoglobin level, erythrocyte count, and IgE serum level. Two months later, results of Baermann and coprocultures for larvae diagnosis were both negative.

Strongyloides stercoralis is one of the few nematodes with the ability to produce autoinfection. The parasite can multiply inside the host, regardless of exogenous contamination, due to transformation of rhabditiform larvae into infective filariforms, leading to persistent infections. However, in most hosts, the course of parasitism remains quiescent, with no significant morbidity. This parasite-host balance can be disrupted under conditions of impaired cellular immunity, resulting in a life-threatening strongyloidiasis (Lam et al., 2006).

In this case, the patient had no gastrointestinal or pulmonary symptoms, despite the very intense discharge of $S$. stercoralis larvae in his stool. He was not under glucocorticoid therapy, tested negative for HTLV and HIV, but had a history of alcohol addiction for more than $20 \mathrm{yr}$

Ethanol intoxication activates the HPA axis, elevating corticosterone levels (Ogilvie et al., 1998; Laszlo et al., 2001; Choudhry and Chaudry, 2006), which in turn suppresses $T$ cell function, decreasing intestinal immunity (Choudhry et al., 2004, 2006). Moreover, corticosterone upregulates IL-18, which enhances myeloperoxidase activity, altering the intestinal barrier function ( $\mathrm{Li}$ et al., 2006). Neutrophils may also play a role in increasing intestinal permeability in acute and chronic ethanol intoxication (Sir et al., 2000; Choudhry et al., 2006). The IL-18mediated increase in chemokines and adhesion molecules is likely to cause intense neutrophil accumulation in the intestinal tissue, contributing to the impairment of intestinal immunity (Choudhry et al., 2006; Li et al., 2007). It has also been suggested that there may be a direct effect of ethanol on the Th2 type of immune response. However, mice receiving an ethanol-containing diet were capable of generating a Th2-protective immune response when infected with Strongyloides sp. (Krolewiecki et al., 2001).

A significant decrease in hemoglobin level in $42 \%$ of patients with disseminated strongyloidiasis has been described by Lam and colleagues (2006). In the case described herein, the patient presented with a hemoglobin level of $4.5 \mathrm{~g} / \mathrm{dl}$. The etiology of anemia in alcoholics is often multifactorial, including poor nutrition, chronic inflammation, blood loss, liver dysfunction, and ineffective erythropoiesis (Lewis et al., 2007). Alcohol can be directly toxic to the bone marrow and, when associated with poor nutrition, frequently causes marked anemia, even if liver disease is absent (Latvala et al., 2004). Studies have shown that many bone marrow biopsy samples from alcoholics are abnormal, mostly showing megaloblastic and/or sideroblastic changes or alterations that suggest iron deficiency (Latvala et al., 2004; Lewis et al., 2007). In addition to direct bone marrow damage, excessive alcohol intake, along with poor diet, can lead to inadequate intestinal absorption of vitamin B12 and folic acid, causing megaloblastic anemia (Lewis et al., 2007). In the present case, the patient had normal levels of both vitamins, ruling out the influence of this mechanism on abnormal erythroblast production. Iron deficiency due to malnutrition, along with intestinal mucosa inflammation exacerbated by hematophagism and bleeding ulcers as result of $S$. stercoralis infection itself, may be the major factors responsible for the intense anemia observed at presentation. In fact, the levels of serum iron and ferritin were both markedly reduced and presence of blood in fecal samples was confirmed.

We should also point out the hemoglobin profile of this patient. Carriers of HbS trait (hemoglobin AS) are not expected to develop hemolytic anemia or other clinical symptoms related to sickle cell disease, except under conditions that favor the process of sickling, such as hypoxia, acidosis, and dehydration (Mura and Ferraz, 2007). Clinical examination and laboratory results did not indicate the presence of any sign of this hematologic pathology.
At presentation, the patient had a discrete elevation of the eosinophil count and a high concentration of IgE. As we did not have previous hematological records, we suppose that the increased eosinophilia and high $\operatorname{IgE}$ level in peripheral blood were a result of the patient's immune response to the parasite. This may have temporarily protected the patient from dissemination of infection, but not controlled the parasite proliferation. In fact, patients with strongyloidiasis and co-infection with HTLV-1, which modifies the immune response toward the Th1 type, have lower levels of serum IgE and of peripheral eosinophil counts, which is frequently associated with dissemination of the parasite (Carvalho and Porto, 2004).

The antibodies IgA, IgE, and IgG4 seem to be more related to modulation of S. stercoralis infection than other immunoglobulins (Atkins et al., 1999; Mota-Ferreira et al., 2009). IgA reduces worm fecundity as well as egg viability, therefore decreasing larvae shedding, IgG4 modulates IgE-mediated response, and $\operatorname{IgE}$ regulates the autoinfection by binding to $\mathrm{Fc}$ receptors on the surface of effector cells, especially mast cells and basophils, inducing degranulation and release of inflammatory mediators (Atkins et al., 1999).

The decrease in $\operatorname{IgA}$ secretion has also been implicated in the high prevalence of $S$. stercoralis infection in alcoholic patients with hepatic cirrhosis (Pelletier et al., 1982). In our patient, the levels of serum IgA, $\mathrm{IgM}$, and $\operatorname{IgG}$ were normal and the level of $\operatorname{IgE}$ was greatly elevated. Despite the normal or elevated levels of immunoglobulin, there was an increase in parasite load. On the other hand, as they were not tested, we cannot rule out either a deficiency in antibody-specific response to helminth antigens or a deficiency in the production of secretory $\operatorname{IgA}$, a very important antibody for intestinal mucosal protection.

Adherence of monocytes and polymorphonuclear cells to filariform larvae after complement activation suggests a role of the complement system in the nonspecific immune defense mechanism against $S$. stercoralis (Messias et al., 1994). In fact, complement is required for protection in both innate and acquired immune response to S. stercoralis (Kerepesi et al., 2006). Complement proteins can function as a chemo-attractant and stimulate degranulation of effector cells, including neutrophils and eosinophils (Gasque, 2004). A study showing that C5 knockout mice sustain the protective immunity to $S$. stercoralis, despite the lack of poreforming membrane attack complex, corroborates the hypotheses that the complement component $\mathrm{C} 3$, in collaboration with IgM and neutrophils, may be major factors responsible for killing larvae (Kerepesi et al., 2006).

Strongyloides stercoralis hyperinfection is an emerging tropical infection that requires greater clinician awareness. Because of the lack of familiarity with this syndrome in healthcare institutions in industrialized countries, physicians may misdiagnose the disease and delay the treatment. The mortality rate is $15 \%$ in hyperinfection syndrome and can reach around $80 \%$ when dissemination to other tissues occurs (Lam et al., 2006; Vadlamudi et al., 2006), which is probably due to the late diagnosis.

Mechanisms underlying $S$. stercoralis hyperinfection are complex and not fully understood. Patients with asymptomatic latent infections may develop severe forms of the disease, triggered by corticosteroid-induced immunosuppression (Lemos et al., 2003; Concha et al., 2005). The role of corticosteroids in hyperinfection can be related to the induction of eosinophil and mast cell depletion in the intestinal mucosa (Soda et al., 1993). In addition to their role as effector cells, eosinophils can present $S$. stercoralis antigens to $\mathrm{T}$ cells and initiate an immune response to the helminth, detected by the increase of expression of CD69, CD86, and MHC class II surface markers (Padigel et al., 2006).

Additional studies are needed to delineate the alterations in intestinal immunity and barrier functions in alcoholic patients that predispose to either infection or hyperinfection by the helminth. The increased levels of endogenous corticosterone and the malnutrition so often observed in ethanol-addicted patients could be implicated in the hyperinfection described herein. In addition, chronic alcohol ingestion can directly change the morphology of intestinal villi and alter intestinal mucosal permeability (Worthington et al., 1978).

The diagnosis of strongyloidiasis is often delayed and overlooked because of the nonspecificity of symptoms. Clinicians in endemic regions must be aware of this when evaluating individuals who abuse ethanol. Latent infections may be a serious medical problem for these patients, potentially leading to fatal cases. Therefore, it is highly recommended that all alcoholics, even those who are asymptomatic, be screened for $S$. stercoralis infection by searching for larvae in stool samples or by detecting specific serum antibodies. 


\section{LITERATURE CITED}

Atkins, N. S., D. J. Conway, J. F. Lindo, J. W. Bailey, and D. A. P. BuNDY. 1999. L3 antigen-specific antibody isotype responses in human strongyloidiasis: Correlations with larval output. Parasite Immunology 21: 517-526.

Carvalho, E. M., And A. F. Porto. 2004. Epidemiological and clinical interaction between HTLV-1 and Strongyloides stercoralis. Parasite Immunology 26: 487-497.

Choudhry, M. A., S. N. Rana, M. J. Kavanaugh, E. J. Kovacs, R. L. Gamelli, And M. M. SAYeed. 2004. Impaired intestinal immunity and barrier function: A cause for enhanced bacterial translocation in alcohol intoxication and burn injury. Alcohol 33: 199-208.

- X. Li, And I. H. Chaudry. 2006. A role for corticosterone in impaired intestinal immunity and barrier function in a rodent model of acute alcohol intoxication and burn injury. Journal of Neuroimmune Pharmacology 1: 428-434.

Concha, R., W. Harrington JR., And A. I. Rogers. 2005. Intestinal strongyloidiasis: Recognition, management, and determinants of outcome. Journal of Clinical Gastroenterology 39: 203-211.

Dada-Adegbola, H. O., And R. A. Bakare. 2004. Strongyloidiasis in children five years and below. West African Journal of Medicine 23: 194-197.

Gaburri, D., A. K. Gaburri, E. Hubner, M. H. Lopes, A. M. Ribeiro, G. A. Paulo, F. H. Pace, P. D. Gaburri, A. T. Ornellas, J. O Ferreira, et AL. 1997. Intestinal parasitosis and hepatic cirrhosis. Arquivos de Gastroenterologia 34: 7-12.

GAsQue, P. 2004. Complement: A unique innate immune sensor for danger signals. Molecular Immunology 41: 1089-1098.

Grove, D. I. 1996. Human strongyloidiasis. Advances in Parasitology 38: 251-309.

Kerepesi, L. A., J. A. Hess, T. J. Nolan, G. A. Schad, and D. Abraham. 2006. Complement component C3 is required for protective innate and adaptive immunity to larval Strongyloides stercoralis in mice. Journal of Immunology 176: 4315-4322.

Kobayashi, J., H. Hasegawa, E. C. Soares, H. Toma, A. R. Dacal, M. C. Brito, A. Yamanaka, A. A. Foli, and Y. Sato. 1996. Studies on prevalence of Strongyloides infection in Holambra and Maceió, Brazil, by the agar plate faecal culture method. Revista do Instituto de Medicina Tropical de São Paulo 38: 279-284.

Krolewiecki, A. J., S. Leon, P. A. Scott, T. J. Nolan, G. A. Schad, and D. Abraham. 2001. Effect of chronic ethanol consumption on protective T-helper 1 and T-helper 2 immune responses against the parasites Leishmania major and Strongyloides stercoralis in mice. Alcoholism: Clinical and Experimental Research 25: 571-578.

Lam, C. S., M. K. Tong, K. M. Chan, and Y. P. Siu. 2006. Disseminated strongyloidiasis: A retrospective study of clinical course and outcome. European Journal of Clinical Microbiology and Infectious Diseases 25: $14-18$.

László, F. A., C. Varga, I. Pávó, J. Gardi, M. Vecsernyés, M. Gálfi, E. Morschl, F. László, and G. B. Makara. 2001. Vasopressin pressor receptor-mediated activation of HPA axis by acute ethanol stress in rats. American Journal of Physiology: Regulatory, Integrative and Comparative Physiology 280: R458-R465.

Latvala, J., S. Parkila, and O. Niemelä. 2004. Excess alcohol consumption is common in patients with cytopenia: Studies in blood and bone marrow cells. Alcoholism: Clinical and Experimental Research 28: 619-624.

Lemos, L. B., Z. Qu, R. Laucirica, And H. L. Fred. 2003. Hyperinfection syndrome in strongyloidiasis: Report of two cases. Annals of Diagnostic Pathology 7: 87-94.

Lewis, G., M. P. Wise, C. Poynton, And A. Godkin. 2007. A case of persistent anaemia and alcohol abuse. Nature Clinical Practice. Gastroenterology and Hepatology 4: 521-526.

Li, X., S. N. Rana, M. G. Schwacha, I. H. Chaudry, and M. A. Choudhry. 2006. A novel role for IL-18 in corticosterone-mediated intestinal damage in a two-hit rodent model of alcohol intoxication and injury. Journal of Leukocyte Biology 80: 367-375.

M. G. Schwacha, I. H. Chaudry, and M. A. Choudhry. 2007. Acute alcohol intoxication potentiates neutrophil-mediated intestine tissue damage following burn injury. Shock 29: 377-383.

Machado, E. R., AND J. M. CostA-CRuZ. 1998. Strongyloides stercoralis and other enteroparasites in children at Uberlândia city, state of Minas Gerais, Brazil. Memórias do Instituto Oswaldo Cruz 93: 161164.

Messias, I. J. T., R. M. Genta, And W. D. Mohren. 1994. Adherence of monocytes and polymorphonuclear cells to infective larvae of Strongyloides stercoralis after complement activation. Journal of Parasitology 80: 267-274.

Mota-Ferreira, D. M., M. R. Gonçalves-Pires, A. F. Júnior, M. C. Sopelete, V. O. Abdallah, and J. M. Costa-Cruz. 2009. Specific $\mathrm{IgA}$ and $\mathrm{IgG}$ antibodies in paired serum and breast milk samples in human strongyloidiasis. Acta Tropica 109: 103-107.

Mura, M., And M. H. C. Ferraz. 2007. Sickle cell trait-heterozygous for the hemoglobin S. Revista Brasileira de Hematologia e Hemoterapia 29: $223-225$.

Ogilvie, K., S. Lee, B. Weiss, And C. Rivier. 1998. Mechanisms mediating the influence of alcohol on the hypothalamic-pituitaryadrenal axis responses to immune and nonimmune signals. Alcoholism: Clinical and Experimental Research 22: 243S-247S.

Oliveira, L. C., C. T. Ribeiro, D. M. Mendes, T. C. Oliveira, and J. M. Costa-CRuz. 2002. Frequency of Strongyloides stercoralis infection in alcoholics. Memórias do Instituto Oswaldo Cruz 97: 119-121.

Padigel, U. M., J. J. Lee, T. J. Nolan, G. A. Schad, and D. Abraham. 2006. Eosinophils can function as antigen-presenting cells to induce primary and secondary immune responses to Strongyloides stercoralis. Infection and Immunity 74: 3232-3238.

Pelletier, G., M. J. Briantais, C. Buffet, J. Pillot, and J. P. Etienne. 1982. Serum and intestinal secretory IgA in alcoholic cirrhosis of the liver. Gut 23: 475-480.

Rossi, C. L., C. D. Partel, A. L. Teixeira, E. E. Takahashi, S. De Barros-Mazon, and L. J. DA Silva. 1993. Strongyloidiasis in Campinas city region (Brazil). Parasitological and serological data. Tropical \& Geographical Medicine 45: 189-190.

Safdar, A., K. Malathum, S. J. Rodriguez, R. Husni, and K. V. Rolston. 2004. Strongyloidiasis in patients at a comprehensive cancer center in the United States. Cancer 100: 1531-1536.

Silva, C. V., M. S. Ferreira, A. S. Borges, and J. M. Costa-Cruz. 2005. Intestinal parasitic infections in HIV/AIDS patients: Experience at a teaching hospital in central Brazil. Scandinavian Journal of Infectious Diseases 37: 211-215.

Sir, O., N. Fazal, M. A. Choudhry, R. L. Gamelli, and M. M. SAyeed 2000. Neutrophil depletion prevents intestinal mucosal permeability alterations in burn-injured rats. American Journal of Physiology: Regulatory, Integrative and Comparative Physiology 278: R1224 R1231.

Soda, K., S. Kawabori, N. Kanai, J. Bienenstock, and M. H. Perdue. 1993. Steroid-induced depletion of mucosal mast cells and eosinophils in intestine of athymic nude rats. International Archives of Allergy and Immunology 101: 39-46.

Szabo, G. 1999. Consequences of alcohol consumption on host defense. Alcohol and Alcoholism 34: 830-841.

Vadlamudi, R. S., D. S. Chi, and G. Krishnaswamy. 2006. Intestinal strongyloidiasis and hyperinfection syndrome. Clinical and Molecular Allergy 4: 8.

Worthington, B. S., L. Meserole, and J. A. Syrotuck. 1978. Effect of daily ethanol ingestion on intestinal permeability to macromolecules. American Journal of Digestive Diseases 23: 23-32.

Zago-Gomes, M. P., K. F. Aikawa, S. F. Perazzio, C. S. Gonçalves, and F. E. Pereira. 2002. Prevalence of intestinal nematodes in alcoholic patients. Revista da Sociedade Brasileira de Medicina Tropical 35: $571-574$. 